\title{
Chinese women's experiences, emotions and expectations of breast-feeding in public: a qualitative study
}

\author{
Ya Zhao, Yan-Qiong Ouyang* and Sharon R Redding \\ School of Health Sciences, Wuhan University, No. 115 Donghu Road, Wuhan 430071, Hubei, People's Republic of China
}

Submitted 27 September 2017: Final revision received 21 November 2017: Accepted 27 November 2017: First published online 1 February 2018

\begin{abstract}
Objective: To explore Chinese mothers' experiences, emotions and expectations of breast-feeding in public places.

Design: Exploratory qualitative study. Purposive sampling was used to recruit participants and face-to-face interviews were conducted. Themes were identified by content analysis.

Setting: Two different geographical communities in Wuhan, Hubei Province, central China, March-May 2016.

Subjects: A total of twenty-seven mothers aged 23-33 years, who had one child under 3 years of age and had experience of breast-feeding in public places.

Results: Seven themes emerged from the interviews: struggling to balance infant's needs and personal feelings; embarrassed or natural emotion regarding breastfeeding in public places; effect of cultural and social norms; internalized concerns going beyond actual social reaction; measures to make breast-feeding in public places easier; desire for more public facilities; and expecting emotional support from society members.

Conclusions: More positive social support, favourable policies and necessary facilities were desired to enable mothers to breast-feed in an appropriate public location. Women expected increased public acceptance of breast-feeding practices and support from government health officials to ensure women's success in breast-feeding in public settings.
\end{abstract}

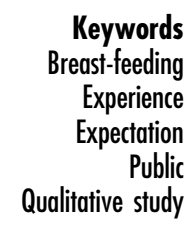

It is well known that breast-feeding, especially exclusive breast-feeding during the first 6 months of an infant's life, has many short- and long-term benefits for mothers, infants and society ${ }^{(1,2)}$. It is recommended that mothers should breast-feed their infants exclusively for the first 6 months and continue up to 2 years of age or beyond with complementary foods ${ }^{(3,4)}$. Mothers should feed babies whenever they want to eat and human milk is widely recommended as the best food for infants ${ }^{(5,6)}$. Despite the many benefits of breast-feeding, the breastfeeding rate is not high. According to UNICEF, the rate of exclusive breast-feeding within the first 6 months in China from 2009 to 2013 was $28 \%^{(7)}$, which is lower than the global rate of $38 \%{ }^{(8)}$.

Inconvenience and embarrassment of breast-feeding in public places may be one reason leading to a low exclusive breast-feeding rate and short breast-feeding duration. Women's experiences of breast-feeding in public places, feelings and decisions have been explored in limited studies $^{(9-11)}$. Most women who have breast-fed in public places have received negative reactions from society members, which resulted in mothers' unpleasant feelings: embarrassed, shy, and even shamed, scared and hated ${ }^{(12,13)}$. Discomfort with experiences of breast-feeding in public places plays an important role in shaping infant feeding choice and the decision to stop breast-feeding. This discomfort may result in mothers switching to bottle-feeding outside the home and early termination of breast-feeding.

Recent articles and events in the news reveal that breastfeeding in public places is not socially accepted. In 2015, a Chinese article entitled 'Beijing subway lactation' presented a photograph of a mother who was breast-feeding in a subway and the caption 'Exposing sexual organs in public areas' was spread nationwide via a popular, microblog platform. This event drew social attention and evoked heated discussion on whether it was proper for mothers to breast-feed their children in public places ${ }^{(14)}$. This was not an isolated incident. A previous paper reported a number of breast-feeding mothers had been treated unfairly and requested to leave a restaurant or coffee shop ${ }^{(12)}$. In the UK, a mother in an upmarket London hotel was asked by members of the public to cover her breasts with a large serviette when feeding her 12-week-old infant ${ }^{(15)}$. Similarly, in the USA, a woman was 
asked to move to the restroom because she was breastfeeding her 6-week-old infant during a shopping trip ${ }^{(16)}$. Some researchers have focused on public attitudes and opinions about breast-feeding in public places and reported that some people were embarrassed, uncomfortable or even offended when facing women breast-feeding in public places such as a shopping centre, bus or subway. They accepted breast-feeding in public places on the condition that mothers did it discreetly ${ }^{(17-19)}$.

In China, exclusive breast-feeding and its barriers have been discussed in a previous study which reported that perceived breast-milk insufficiency was the most important reason for giving up exclusive breast-feeding ${ }^{(20)}$. Meanwhile, it was reported that fathers played an important role in improving the exclusive breast-feeding rate ${ }^{(21)}$. Another study conducted in Shanghai, China, found that a theory-based breast-feeding promotion intervention was an effective measure to improve exclusive breastfeeding ${ }^{(22)}$. However, no published data on breastfeeding in public places from the mother's perspective in the Chinese context have been found to date. A better understanding of mothers' breast-feeding experiences in public settings is needed, to provide them with assistance and support. The present qualitative study explored the experiences of breast-feeding mothers in public places in Wuhan, China. Their expectations regarding breastfeeding in public places are also discussed.

\section{Materials and methods}

\section{Design}

This was an exploratory qualitative study exploring the experience and expectations of breast-feeding in public places from Chinese women's perspectives using purposive sampling. Semi-structured face-to-face interviews were conducted.

\section{Participants}

The study took place in Wuhan, the capital city of Hubei Province, central China. Women were recruited from health-care centres in two different geographical communities during their infant's check-up. Participants met the following criteria: (i) had one child under 3 years of age; (ii) had experience of breast-feeding in public places; and (iii) were able to speak and understand Mandarin Chinese. A total of twenty-seven participants with varying backgrounds were interviewed. No new concepts emerged after twenty-four participants were interviewed. The researchers then continued to interview three participants who repeated concepts that emerged from the previous interviews, representing data saturation and completion of data collection.

\section{Process}

Women were contacted directly by the researcher. They were offered an introduction and explanation about the purpose of the research to discuss breast-feeding experiences and seek suggestions. If a woman expressed interest, information was provided verbally and in writing as to how the interview would be conducted prior to obtaining signed informed consent. An appointment was made to do the interview in a separate room at the community health centre or in the participant's home at her convenience. A topic guide, based on relevant literature and the aims of the study, was used to facilitate each interview and focused on: women's experiences of breast-feeding in public places; women's attitudes and beliefs towards breast-feeding in public places; women's feelings about breast-feeding and response of people in the public setting; measures accessible to make breast-feeding women and other people feel more comfortable; desired support to make breast-feeding in public places successful; and other comments related to the experience. Participants were encouraged to speak freely. The interview was audio-taped after obtaining written informed consent of participants and they were given a small gift in appreciation of their participation. Interviews, which averaged 30-45 min, were conducted from March to May 2016.

\section{Data analysis}

Data were collected and analysed simultaneously. Content analysis was used to identify major themes and subthemes relating to breast-feeding in public places. The analysis followed the methods of Elo and Kyngas ${ }^{(23)}$.

The process of analysis began with two researchers transcribing the data into text, word for word, and reading and re-reading the transcriptions. The transcripts were returned to the participants to clarify misinterpretations or missing information. Units of analysis (such as a letter, word, sentence, portion of pages, or words that related to women's experience of breast-feeding in public) were then selected by two researchers separately and compared. A set of codes or labels for related groups of data was identified. Notes and headings were inserted in the text during reading. The material was re-read, and as many headings as necessary were noted in the margins to describe all aspects of the content. A list of significant statements was developed about mothers' reactions to the situation when their infants required breast-feeding in public. From these statements, a list of non-repetitive, nonoverlapping statements was synthesized. Significant statements were then grouped into larger units of information. The aim was to reduce the number of categories by collapsing those that were similar or dissimilar into broader higher-order categories. From this, a description of women's experiences and expectation of breast-feeding in public places was written.

\section{Ethics principles}

The study was approved by the Research Ethics Committee of Wuhan University. Procedures were followed to 
maintain confidentiality and participants' names were not attached to transcripts or any reports or publications. Participation was voluntary, all participants provided written informed consent, and withdrawal from the study resulted in no prejudice.

\section{Results}

A total of twenty-seven participants aged 23-33 years were interviewed. Monthly income of most participants (15/27) was less than 5000 Renminbi (RMB). Of twenty-seven participants, twelve graduated from high school, eleven women had a bachelor's degree and four obtained a master's degree. Ten were not employed prior to pregnancy or resigned upon becoming pregnant. Duration of any breast-feeding ranged from 3 to 15 months. Further information on the participants is shown in Table 1.

Seven main themes emerged from the interviews, as follows.

\section{Theme 1: Struggling to balance infant's needs and personal feelings}

In general, women tended to find a balance between their own feelings, their infant's needs and the reactions of other people. Since different women had different priorities, they behaved differently when their babies needed to be fed outside the home. They were identified as babycentred women or mother-centred women.

In most cases, baby-centred women believed the highest priority was their infants, and this surpassed all personal needs. Therefore, they would breast-feed without hesitation. Because infants require feeding every 2 to $3 \mathrm{~h}$, breast-feeding mothers were likely to feed infants in public places unless mothers stayed at home most of the time. When the infant was hungry, most of the mothers would breast-feed whenever and wherever they were at that time. They believed that 'breast milk is the best for infants', 'the infants felt warm and placated when breastfed' and 'breast-feeding offered security and protection'. They wanted to provide ideal nutrients. Some women ( $n$ 5) thought 'it was a symbol of maternal love'. They had no qualms about breast-feeding since feeding infants when needed was a mother's responsibility and they were doing the right thing for their infants:

'There is no doubt that I should feed my baby when he was hungry. Babies are quite different from adults who could endure starvation. I must give him breast milk when he cries.'

'Breast milk is best. Breast-feeding is normal. I must feed my baby when she is hungry. Mother's love is great and selfless. I should make my baby feel warm and safe.'

Some mother-centred women thought it was inconvenient and shameful to breast-feed outside the home ( $n$ 8).
These women took the unpleasant feeling as their priority, so they avoided breast-feeding in public places as much as possible. They made a feeding plan at home before going out. After finishing their affairs, they would rush to return home. Otherwise, they would feed the baby with water, or distract the child's attention with toys or pacifiers. Some women took formula with them so that they could avoid the inconvenience of breast-feeding in public places. They would feed their babies only if there were no other alternatives. Even though they finally resorted to breast-feeding, they described it as 'cheeky', 'an intolerable mood' and 'the last choice':

'I tried my best not to breast-feed outside home. Sometimes there were no other choices when infants need to eat, so I breast-fed in public places with cheekiness.'

\section{Theme 2: Embarrassed or natural emotion regarding breast-feeding in public places}

Although most women stated that they considered breastfeeding in public a normal and common thing and they also were willing to overcome difficulties, they still felt embarrassed, uncomfortable, shy or even scared. In most cases, they thought breast-feeding could occur in private places and should not be done in the presence of others, even friends or family members, especially when there were men around:

'I feel unnatural and I am unwilling to do this [breastfeeding in public places]. At beginning, I was very shy to do this. I dared not to feed him [my baby] outside home. So, I gave him a pacifier when he cried and I put it in his mouth.'

'I think it is ashamed to do it [breast-feeding]. It is very embarrassing to open clothes and expose my breasts. It is scary that other people peer at my breasts.'

A few women felt normal and natural without any discomfort when breast-feeding in public places. They believed that breast-feeding was a natural thing, just as adults need to eat when they were hungry. Babies are born to be fed with mothers' breast milk. It is what means to be a mother. Some women thought it was a great thing to breast-feed infants and they felt happy with this activity. Some women commented on the nutritional benefits for infants and the immune properties of breast milk, while others stated it was an inherent and instinctive process to breast-feed. These mothers mentioned that they did not care what other people thought and paid no attention to the response of people in their surroundings. They also believed that other people could understand the behaviour of breast-feeding in public places:

'I think everyone can understand this [breast-feeding] because we all were fed by breast milk and 
Table 1 Characteristics of the study participants: mothers aged 23-33 years, who had one child under 3 years of age and experience of breast-feeding in public places, Wuhan, Hubei Province, central China, March-May 2016

\begin{tabular}{|c|c|c|c|c|c|c|c|}
\hline$\#^{*}$ & $\begin{array}{c}\text { Age } \\
\text { (years) }\end{array}$ & $\begin{array}{l}\text { Education } \\
\text { level }\end{array}$ & $\begin{array}{l}\text { Family income/month } \\
\text { (Renminbi, RMB) }\end{array}$ & Employment & $\begin{array}{l}\text { Breast-feeding } \\
\text { duration (months) }\end{array}$ & $\begin{array}{l}\text { Breast-feeding model } \\
\text { within } 6 \text { months }\end{array}$ & $\begin{array}{l}\text { Infant's age } \\
\text { (months) }\end{array}$ \\
\hline 1 & 23 & $\begin{array}{l}\text { High } \\
\text { school }\end{array}$ & $<5000$ & No & 8 & Exclusively & 8 \\
\hline 2 & 26 & Bachelor & $5000-8000$ & Yes & 4 & Exclusively & 4 \\
\hline 3 & 24 & $\begin{array}{l}\text { High } \\
\text { school }\end{array}$ & $<5000$ & No & 6 & Exclusively & 6 \\
\hline 4 & 31 & Bachelor & 5000-8000 & Yes & 6 & Mixed & 6 \\
\hline 5 & 28 & Master & $>8000$ & Yes & 12 & Mixed & 12 \\
\hline 6 & 26 & Bachelor & $<5000$ & Yes & 10 & Mixed & 18 \\
\hline 7 & 30 & Master & $5000-8000$ & Yes & 8 & Mixed & 8 \\
\hline 8 & 27 & Bachelor & $<5000$ & No & 4 & Exclusively & 4 \\
\hline 9 & 25 & Bachelor & $<5000$ & No & 6 & Exclusively & 6 \\
\hline 10 & 36 & $\begin{array}{l}\text { High } \\
\text { school }\end{array}$ & 5000-8000 & Yes & 6 & Mixed & 6 \\
\hline 11 & 26 & Bachelor & $>8000$ & Yes & 12 & Mixed & 12 \\
\hline 12 & 22 & $\begin{array}{l}\text { High } \\
\text { school }\end{array}$ & $<5000$ & No & 4 & Exclusively & 4 \\
\hline 13 & 29 & Bachelor & $<5000$ & No & 8 & Mixed & 8 \\
\hline 14 & 25 & Bachelor & $5000-8000$ & Yes & 6 & Mixed & 8 \\
\hline 15 & 27 & $\begin{array}{l}\text { High } \\
\text { school }\end{array}$ & $>8000$ & Yes & 3 & Exclusively & 3 \\
\hline 16 & 29 & $\begin{array}{l}\text { High } \\
\text { school }\end{array}$ & $<5000$ & Yes & 10 & Mixed & 24 \\
\hline 17 & 31 & Master & $5000-8000$ & Yes & 15 & Mixed & 18 \\
\hline 18 & 25 & $\begin{array}{l}\text { High } \\
\text { school }\end{array}$ & $<5000$ & No & 6 & Exclusively & 6 \\
\hline 19 & 24 & Bachelor & $>8000$ & Yes & 3 & Exclusively & 3 \\
\hline 20 & 29 & $\begin{array}{l}\text { High } \\
\text { school }\end{array}$ & $5000-8000$ & Yes & 8 & Mixed & 8 \\
\hline 21 & 23 & $\begin{array}{l}\text { High } \\
\text { school }\end{array}$ & $<5000$ & No & 12 & Mixed & 12 \\
\hline 22 & 33 & $\begin{array}{l}\text { High } \\
\text { school }\end{array}$ & $<5000$ & Yes & 10 & Mixed & 36 \\
\hline 23 & 31 & $\begin{array}{l}\text { High } \\
\text { school }\end{array}$ & 5000-8000 & No & 12 & Exclusively & 12 \\
\hline 24 & 27 & Master & $<5000$ & Yes & 8 & Mixed & 8 \\
\hline 25 & 23 & $\begin{array}{l}\text { High } \\
\text { school }\end{array}$ & $<5000$ & Yes & 10 & Mixed & 10 \\
\hline 26 & 25 & Bachelor & $<5000$ & Yes & 6 & Mixed & 6 \\
\hline 27 & 25 & Bachelor & $<5000$ & No & 10 & Exclusively & 12 \\
\hline
\end{tabular}

*\# means participant number.

grew up. Formula is the last choice when mothers' milk is insufficient.'

'I don't care about other people's thoughts, and I just want to feed my baby. It's a mother's responsibility to feed infants with breast milk.'

\section{Theme 3: Effect of cultural and social norms}

A few mothers mentioned that they did not want or dare to breast-feed in public because of Chinese traditional cultural and social norms. In ancient China, women exposing the skin below the neck were considered dissolute. Traditional Chinese culture also pays much attention to the sexual role of breasts, and many women said that exposing their breasts was not acceptable in public places. Discussion of sex is a taboo subject in Chinese culture, let alone exposing breasts and genitalia. This has been a custom established by the people through long social practice. Thus, some women stated that exposing their breasts when breast-feeding in public places disobeyed society's rules and was somewhat uncivilized. They were worried about the moral judgement of others:

'Since I was a child, I was told that exposing breasts in front of others was indecent. Of course, I think in public places lactation women should use something to cover or hide their breasts. Even so, I think that women had better not breast-feed in public places.'

'A person with high quality will be conscious of their image ... [breast-feeding in public places] is related to a person's quality and personality. Maybe my opinions are a little traditional and my thought is conservative, so I think it [breast-feeding in public places] should be a private behaviour. I don't want others think that I am inelegant. It is the respect for others. After all, not all people can accept this behaviour...' 


\section{Theme 4: Internalized concerns going beyond actual social reaction}

A large number of mothers worried about other people's response and were afraid of bad remarks by society members, such as being shameless and uncivilized. However, when asked about negative remarks by other people, few women admitted receiving derogatory comments or behaviours from others. Some women mentioned the 'Beijing subway lactation' ${ }^{\text {(14) }}$ news article and they were afraid that something similar could happen to them, so they tried their best to not breast-feed in public places. It seems that women's worries were more internalized than overt. They were worried about other people's perspectives when among strangers, especially men. One woman was feeding her baby when interviewed. She said it was okay to breast-feed in front of females (the researcher is female), but if they were males, she would avoid doing so. Three participants admitted that they would breast-feed in a public place if the people around were family members or female friends, but they would abandon breast-feeding when there were strangers:

'Someone stare at me with unusual facial expression

... I don't want others to think that I am inelegant ...

so I try to avoid breast-feeding in public places.'

'I've never met anyone who's been telling me what to do when I breast-fed in public places, but some looked at me strangely. I think they might believe that I'm morally deficient.'

\section{Theme 5: Measures to make breast-feeding in public places easier}

Some women were persistent in their breast-feeding practices, but they did not want to expose their body when breast-feeding in public places. They tried to compromise between their infant's needs and their own feelings. They took measures to make themselves and others around them feel more comfortable. These measures included going to a location with lactation rooms and using a nursing cover, scarf, clothes or umbrella to hide their body. If nothing was available, they would ask family members to create a barrier by standing in front of them in order that strangers could not see them breast-feeding. Additionally, they would turn their face to the wall or try their best to find a corner with few people, so they could breast-feed their babies:

'I usually bring a small blanket or clothes when going out with my baby so I can use this to cover my breast when breast-feeding in public. I also tried best to find somewhere with nursing rooms or with few people.'

'I usually find a corner and turn my face to the wall when breast-feeding in public. What's more, I just expose my nipple outside and it was in my baby's mouth, so others cannot see my breasts.'
'At first, I didn't get used to breast-feeding in public places, so I brought a pacifier. When she [her baby] cries, I put it in her mouth. Sometimes I use a toy to attract her attention or feed her with some water. Later I changed my mind, but I still use clothes to hide my breast when breast-feeding.'

\section{Theme 6: Desire for more public facilities}

In discussion with participants, most women desired more appropriate facilities for breast-feeding in public places. Only three mothers had used lactation rooms in an airport or supermarket. One participant said that she could not find a lactation room in a subway station and, in another location, she found a sign indicating a lactation room; however, it turned out to be a deserted storage area. Some of the mothers agreed that a single room open only for mothers was appropriate and this was the most basic requirement: 'We don't need much. A small separate room and a chair on which I can sit, that's enough.' Sometimes they did not want to breast-feed in public places but there were no suitable facilities. They preferred that the room should have adequate space and privacy to protect infants from noise, sunlight and crowds of people, thus providing security and comfort for both the mother and infant. It was better to have a room with a comfortable chair for good positioning and latching on, to ensure successful breastfeeding outside home:

'I was worried about someone interrupting me when I breast-feed my baby. A single room is better. But I don't know where and how to find it. I have never seen a lactation room in the public places. Have you seen it?'

'I felt that it was the responsibility of society and government. The saying "we should build more lactation rooms" has been said repeatedly for so long time, why I can't see a lactation room?'

\section{Theme 7: Expecting emotional support from society members}

Participants felt it was necessary to be emotionally supported by society members when they were breastfeeding. They hoped that people would be kind and friendly and not say bad words or give weird looks. They hoped that people understood the difficulty of the mothers and avoided staring or discourteous behaviour. Two women mentioned that breast-feeding education including benefits and advantages for mothers and infants should be provided to the general public, especially to young men and women, so that breast-feeding could be more socially acceptable:

'I hope the public can understand that breast-feeding is normal and natural. It is not an uncivilized thing. So please eliminate discrimination and give us some 
support. I try not to bother others, also hope that other people don't point fingers at me.'

\section{Discussion}

The present study highlighted maternal experiences, emotions and expectations of breast-feeding in public places from Chinese mothers' perspectives. In our study, most women thought that breast-feeding in public was natural while a few argued that breast-feeding should occur in a private place when recalling their experience of breast-feeding in public places. Although most women regarded breast-feeding in public as natural, common and unavoidable, some felt embarrassed and shy. Thomson et $a l .{ }^{(24)}$ found that breast-feeding mothers might feel shame, humiliation and fear of criticism which reveals a conflict between their feelings of embarrassment and a desire to do the best for their babies.

According to the theory of planned behaviour ${ }^{(25)}$, personal behaviour is not only determined by attitude, but also affected by social pressure and perceived behavioural control. In the current study, most women stated that social acceptance and civil friendship were needed for mothers to breast-feed in public places. Mothers expected that society members could provide emotional support. Participants in other studies also agreed that a supportive community was very important for breast-feeding ${ }^{(17,26,27)}$. Previous studies showed that some society members had a negative response to breast-feeding in public places. Zhao et $a l .{ }^{(28)}$ investigated Chinese adults and found that $47 \%$ of respondents believed that viewing women breast-feeding in public was embarrassing. In Boyer's ${ }^{(12)}$ study conducted in the UK, almost half of the mothers had been treated unfairly and they were asked to leave a restaurant or coffee shop. Some people felt embarrassed or even offended when seeing breast-feeding in public places ${ }^{(19)}$. Notably, a survey showed that only $43 \%$ of US adults believed women had the right to breast-feed in public places and $72 \%$ thought it was inappropriate to show a woman breast-feeding her baby on television programmes ${ }^{(29)}$. Some people thought that women breastfeeding in public places should be discreet ${ }^{(9)}$. Shortt et al. ${ }^{(11)}$ also reported women felt social pressure to keep breastfeeding hidden from public gaze. Moreover, there was a gap between mothers' positive attitude towards breast-feeding in public places and their perception of negative social norms. Social and medical stressors affect breast-feeding duration $^{(30)}$. It was interesting that no mothers in the present study had received rude or negative behaviours or comments from people. Participants were more worried about social censure reported by social media rather than having actually experienced it. It seems that the decision to breastfeed in public is beyond their own control.

In the present study, participants were worried that breast-feeding in public places would be seen as uncivilized and shameless. Chinese Confucianism required that women wear and dress properly so that they should not expose their skin and curves ${ }^{(31)}$. Public perception is more open today than in the past, but people remain influenced by Confucianism, which results in a contradictory attitude towards breast-feeding in public places. On the one hand, people consider breast-feeding in public as natural and common and should be supported. On the other hand, their attitude is that breast-feeding women should not expose breasts in public places ${ }^{(28)}$. Studies found that cultural support for breast-feeding has a significant effect on mother's choice ${ }^{(32,33)}$. There is a similar situation in the UK where breast-feeding in public places has also not been socially accepted ${ }^{(34)}$. Furthermore, the viewing of breasts as sexual organs is another reason women themselves and others around feel embarrassed. In the present study, a few mothers mentioned that they would cover their body and avoid breast-feeding in the presence of men. Gender perceptions regarding social space affect breast-feeding practices and this has been observed in other cultures ${ }^{(10,19)}$.

Despite long-standing recommendations for the provision of lactation rooms in public places, participants, for the most part, reported that they seldom found a lactation room when needed. The present study revealed social embarrassment and the lack of lactation rooms acted as a barrier to breastfeeding in public places. Only three of the twenty-seven participants had made use of lactation rooms and they reported intense dissatisfaction with the rooms being dirty and disorderly. This implies that it is not enough to merely provide lactation rooms, but they also need proper maintenance. Inconvenience or discomfort with breast-feeding in a public place was an important barrier regarding mothers' choice and duration of breast-feeding. Almost all participants were in agreement that well-managed lactation rooms are needed. They hoped there would be more breast-feeding places in public spaces so that breast-feeding could be more acceptable for both mothers and the public. The Chinese government has made some efforts to solve this problem. In 2013, UNICEF and the Chinese Center for Disease Control and Prevention introduced '10 Square Meters of Love', a campaign aimed at establishing lactation rooms in workplaces and public buildings in China ${ }^{(35)}$. According to a guideline released by the National Health and Family Planning Commission, all major public places in China, such as transportation hubs, large shopping malls and hospitals, should be equipped with mother-and-child facilities used for breast-feeding or diaper changing by the end of $2020^{(36)}$.

Notably, mothers in the current study who reported their experience of breast-feeding in public places with a positive perception usually opted for exclusive and optimum breastfeeding for their infants, and the duration of breast-feeding was longer ${ }^{(37)}$. It has been confirmed that a positive social attitude towards breast-feeding in public spaces was helpful to promote breast-feeding ${ }^{(19)}$. Because of the impact of traditional culture and social opinion as well as the 
inconvenience of breast-feeding in public places, some mothers would bring breast-milk substitute when going out. The same situation was reported in other studies ${ }^{(13,34)}$. This suggests that efforts from many perspectives are needed to support breast-feeding, including government programmes, society members and mothers themselves.

\section{Strengths and limitations}

Based on the perceptions and meanings assigned by people, qualitative research assumes that truth and facts are subjective. Qualitative data promote a richer and deeper description of phenomena, such as an individual's perceptions, attitudes, meanings and overall experiences, than a simplistic questionnaire survey. Therefore, the current study presents the following advantages: conducting a qualitative investigation of women's experiences of breast-feeding in public places and utilizing a qualitative methodology allows for the collection and analysis of mothers' experiences, emotions and expectations of breast-feeding in public places extensively and in depth. A better understanding of mothers' experiences in public settings is helpful for government officials to make policies in China and to promote improved societal attitudes towards breast-feeding in public places.

However, some limitations of qualitative research should also be acknowledged. First, the number of participants in qualitative studies tends to be smaller. Second, participants were recruited purposively rather than randomly, and the influence of investigators' personal values and attitudes on the reporting of findings are potential limitations. In addition, the voluntary participation of mothers may be due to their high level of interest. The present study was conducted in one urban area in China, so caution is needed in generalizing the results.

\section{Conclusion}

A mother's experience of breast-feeding in public places is affected by meeting the needs of her infant, her personal feelings and the response of society. Inconvenience and embarrassment are key factors influencing women who choose to breast-feed in public places. To improve this situation, the Chinese government and health professionals should advocate for improving services in public places for breast-feeding women. Universities, hospitals and communities should provide public breast-feeding education for members of society so as to promote awareness of the benefits of breast-feeding and to change people's deep-rooted traditional concepts, and thus support breast-feeding women.

\section{Acknowledgements}

Acknowledgements: The authors would like to thank the participants and staff of the health-care centres for their support. Financial support: This research received no specific grant from any funding agency in the public, commercial or not-for-profit sectors. Conflict of interest: The authors declared no potential conflicts of interest with respect to the research, authorship and/or publication of this article. Authorship: Y.Z. contributed to research design, data collection, data analysis and manuscript writing. Y.-Q.O. contributed to project development, data analysis and manuscript revision. S.R.R. contributed to manuscript revision and review. Ethics of buman subject participation: The Research Ethics Committee of Wuhan University approved the study. Procedures were followed to maintain confidentiality and all participants provided written informed consent. Participation was voluntary and could be withdrawn at any time without prejudice.

\section{References}

1. Victora CG, Bahl R, Barros AD et al. (2016) Breastfeeding in the 21st century: epidemiology, mechanisms, and lifelong effect. Lancet 387, 475-490.

2. Rollins NC, Bhandari N, Hajeebhoy N et al. (2016) Why invest, and what it will take to improve breastfeeding practices? Lancet 387, 491-504.

3. World Health Organization (2003) Global Strategy for Infant and Young Child Feeding. http://www.who.int/nutrition/ topics/global_strategy/en/(accessed May 2016).

4. Dieterich CM, Felice JP, O'Sullivan E et al. (2013) Breastfeeding and health outcomes for the mother-infant dyad. Pediatr Clin North Am 60, 31-48.

5. American Academy of Pediatrics (2012) Breastfeeding and the use of human milk. Pediatrics 129, e827-e841.

6. World Health Organization (2016) 10 Facts on Breastfeeding. http://www.who.int/features/factfiles/breastfeeding/ facts/en/ (accessed December 2016).

7. UNICEF (2013) China. Statistics. http://www.unicef.org/ infobycountry/china_statistics.html\#114 (accessed June 2016).

8. World Health Organization (2015) Breastfeeding: Global Targets 2025. http://www.who.int/nutrition/globaltarget-2025/infographic_breastfeeding.pdf?ua=1 (accessed December 2015).

9. Boyer K (2011) 'The way to break the taboo is to do the taboo thing', breastfeeding in public and citizen-activism in the UK. Health Place 17, 430-437.

10. Smyth L (2008) Gendered spaces and intimate citizenship, the case of breastfeeding. Eur J Women Stud 15, 83-99.

11. Shortt E, McGorrian C \& Kelleher C (2013) A qualitative study of infant feeding decisions among low-income women in the Republic of Ireland. Midwifery 29, 453-460.

12. Boyer K (2012) Affect, corporeality and the limits of belonging: breastfeeding in public in the contemporary UK. Health Place 18, 552-560.

13. Forster DA \& McLachlan HL (2010) Women's views and experiences of breast feeding: positive, negative or just good for the baby? Midwifery 26, 116-125.

14. China Daily (2015) Is public breastfeeding appropriate? http://www.chinadaily.com.cn/opinion/2015-12/08/content_ 22657039.htm (accessed May 2016).

15. Billock J (2014) London hotel under fire after telling breastfeeding mother to cover up. http://www.flyertalk. com/articles/london-hotel-under-fire-after-telling-breastfeeding -mother-to-cover-up.html/ (accessed December 2016).

16. Lemon K (2014) School worker tells breastfeeding mom to move to bathroom. http://www.wsoctv.com/news/local/ 
school-worker-tells-breastfeeding-mom-move-bathroo/113 357613 (accessed December 2016).

17. Meng X, Daly A, Pollard CM et al. (2013) Community attitudes toward breastfeeding in public places among Western Australia adults, 1995-2009. J Hum Lact 29, 183-189.

18. Mulready-Ward C \& Hackett M (2014) Perception and attitudes: breastfeeding in public in New York City. J Hum Lact 30, 195-200.

19. Spurles PK \& Babineau J (2011) A qualitative study of attitudes toward public breastfeeding among young Canadian men and women. J Hum Lact 27, 131-137.

20. Ouyang YQ, Su M \& Redding SR (2016) A survey on difficulties and desires of breastfeeding women in Wuhan, China. Midwifery 32, 19-24.

21. Su M \& Ouyang YQ (2016) Father's role in breastfeeding promotion: lessons from a quasi-experimental trial in China. Breastfeed Med 11, 144-149.

22. Gu Y, Zhu Y, Zhang Z et al. (2016) Effectiveness of a theorybased breastfeeding promotion intervention on exclusive breastfeeding in China: a randomised controlled trial. Midwifery 42, 93-99.

23. Elo S \& Kyngas H (2008) The qualitative content analysis process. J Adv Nurs 62, 107-115.

24. Thomson G, Ebisch-Burton K \& Flacking R (2015) Shame if you do - shame if you don't: women's experiences of infant feeding. Matern Child Nutr 11, 33-46.

25. Ajzen I \& Fishbein M (1980) Understanding Attitudes and Predicting Social Behavior. Englewood Cliffs, NJ: PrenticeHall.

26. Brown A, Raynor P \& Lee M (2011) Young mothers who choose to breastfeed: the importance of being part of a supportive breast-feeding community. Midwifery $\mathbf{2 7}$, 53-59.

27. Chang SM, Rowe J \& Goopy S (2014) Non-family support for breastfeeding maintenance among career women in Taiwan: a qualitative study. Int $J$ Nurs Pract 20, 293-301.
28. Zhao Y, Ouyang YQ \& Redding SR (2017) Attitudes of Chinese adults to breastfeeding in public: a web-based survey. Breastfeed Med 12, 316-321.

29. Li R, Hsia J, Fridinger F et al. (2004) Public beliefs about breastfeeding policies in various settings. J Am Diet Assoc 104, 1162-1168.

30. Purdy IB, Singh N, Le C et al. (2012) Biophysiologic and social stress relationships with breast milk feeding pre- and post-discharge from the neonatal intensive care unit. J Obstet Gynecol Neonatal Nurs 41, 347-357.

31. Qi Y \& Chi R (2013) The study of Chinese traditional costume under the influence of the Confucian ethical code culture. JJinan Univ (Soci Sci Ed) 23, 84-87.

32. Bailey C, Pain RH \& Aarvold JE (2004) A 'give it a go' breastfeeding culture and early cessation among low-income mothers. Midwifery 20, 240-250.

33. Walburg V, Goehlich M, Conquet M et al. (2010) Breast feeding initiation and duration: comparison of French and German mothers. Midwifery 26, 109-115.

34. Morris C, Zaraté de la Fuente GA, Williams CE et al. (2016) UK views toward breastfeeding in public: an analysis of the public's response to the Claridge's incident. J Hum Lact 32, 472-480.

35. UNICEF \& Chinese Center for Disease Control and Prevention, Maternal and Child Health Care Center (2013) 10 Square Meters of Love. http://www.10m2.unicef.cn/ (accessed May 2016).

36. National Health and Family Planning Commission (2016) Guidance on accelerating the construction of maternal and infant facilities. http://www.moh.gov.cn/jczds/s3577/2016 11/7de90996a6ec4ffa83dd538591c8e7bd.shtml (accessed December 2016).

37. Nnebe-Agumadu UH, Racine EF, Laditka SB et al. (2016) Associations between perceived value of exclusive breastfeeding among pregnant women in the United States and exclusive breastfeeding to three and six months postpartum: a prospective study. Int Breastfeed J 11, 8. 